\title{
Acknowledgement to Reviewers of Beverages in 2015
}

\author{
Beverages Editorial Office \\ Published: 22 January 2016 \\ MDPI AG, Klybeckstrasse 64, CH-4057 Basel, Switzerland; beverages@mdpi.com
}

The editors of Beverages would like to express their sincere gratitude to the following reviewers for assessing manuscripts in 2015.

We greatly appreciate the contribution of expert reviewers, which is crucial to the journal's editorial decision-making process. Several steps have been taken in 2015 to thank and acknowledge reviewers. Good, timely reviews are rewarded with a discount off their next MDPI publication. By creating an account on the submission system, reviewers can access details of their past reviews, see the comments of other reviewers, and download a letter of acknowledgement for their records. This is all done, of course, within the constraints of reviewer confidentiality. Feedback from reviewers shows that most see their task as a voluntary and mostly unseen work in service to the scientific community. We are grateful to our reviewers for the contribution they make.

Allman-Farinelli, Margaret
Amedeo, Maizza
Arbona, Vicent
Ariel Ochoa, N.
Bai, Jinhe
Bain, Robert E.S
Bennett, James
Bhandari, Bhesh
Boatto, Vasco
Bodelón, Oscar G.
Bonaccorsi, Ivana
Bowtell, Joanna L
Caballero, Isabel
Campbell, Ben
Caporaso, Nicola
Chabrol, Didier
Chambers, Edgar, IV
Chandrapala, Jayani
Christova-Bagdassarian, V.
Cran, Marlene J.
Davis, Aaron P.
de Franca Doria, Miguel
di Cristo, Cristiana
Durazzo, Alessandra
Effertz, Tobias
Engelhardt, Ulrich H.
Escudero, Ana
Fell, Jim
Ferrer, Emilia
Franks, Amy M
Giacalone, Davide

Goldfarb, Michael
Gonzalez-Feliu, Jesus
Graham, Dan J.
Granato, Daniel
Habtemariam, Solomon
Hamon, Perla
Harbertson, James F.
Harrington, Robert J.
Heberger, Karoly
Heijungs, Reinout
Heimler, Daniela
Herrmann-Pillath, Carsten
Kao-Walter, Sharon
Kerr, William L.
Ladaru, Georgiana-Raluca
Langowski, Horst-Christian
Lawrence, G.
Lidon, Fernando Cebola
Lipshultz, Steven E.
Marion, Jason W.
Mirosa, Miranda
Montanari, Luigi
Monzó, J. Martínez
Morris, Jonathan
Mulinacci, Nadia
Muñuzuri, Jesús
Nieman, David C
Nieuwoudt, Helene
Oberfeld, Daniel
Österberg, Esa L.
Owusu-Apenten, Richard

Goldfarb, Michael

Gonzalez-Feliu, Jesus

Habtemariam, Solomon

amon, Perla

Harrington, Robert J.

Heberger, Karoly

Heijungs, Reinout

Kao-Walter, Sharon

Langowski, Horst-Christian

Lipshultz, Steven E.

Marion, Jason W.

Monzó, J. Martínez

Morris, Jonathan

Mulinacci, Nadia

Muñuzuri, Jesús

Nieman, David C

Nieuwoudt, Helene

Österberg, Esa L.

Owusu-Apenten, Richard

\author{
Özyürek, Mustafa \\ Palacios, Antonio \\ Parisi, Salvatore \\ Pinto, Rute \\ Poncet, Valérie \\ Pons, Antoni \\ $\mathrm{R}$ Jaeger, Sara \\ Ranadheera, C. Senaka \\ Režek Jambrak, Anet \\ Roberts, Susan \\ Rodríguez-Lázaro, David \\ Rossi, Filippo \\ Rufián Henares, José Ángel \\ Rundh, Bo \\ Salafranca Lázaro, Francisco J. \\ Seff, James M. \\ Severini, C. \\ Simirgiotis, Mario J. \\ Singh, I. \\ Smrke, Samo \\ Stolzenbach, Sandra \\ Strazza, C. \\ Suklje, Katja \\ Taylor, Christopher \\ Terry-McElrath, Yvonne \\ Tomlinson, Philip R. \\ Twede, Diana \\ ur Rahman, Saleem \\ Valzacchi, Sandro \\ Vanderroost, Mike \\ Vrček, Ivana Vinković
}


Beverages 2016, 2, 3

Warriner, Keith

Williams, Ashley Rhoderick
Ying, Danyang

Zabaras, Dimitrios
Zamora, María C.

(C) 2016 by the author; licensee MDPI, Basel, Switzerland. This article is an open access article distributed under the terms and conditions of the Creative Commons by Attribution (CC-BY) license (http://creativecommons.org/licenses/by/4.0/). 